\title{
Neutrophil-to-lymphocyte ratio as an early marker of outcomes in patients with advanced non-small-cell lung cancer treated with nivolumab
}

\author{
Aya Nakaya ${ }^{1}$ (D) Takayasu Kurata $^{1} \cdot$ Hiroshige Yoshioka ${ }^{1} \cdot$ Yuki Takeyasu $^{1} \cdot$ Maiko Niki $^{1} \cdot$ Kayoko Kibata $^{1}$. \\ Naoko Satsutani ${ }^{1} \cdot$ Makoto Ogata $^{1} \cdot$ Takayuki Miyara $^{1} \cdot$ Shosaku Nomura ${ }^{1}$
}

Received: 22 October 2017 / Accepted: 7 February 2018 / Published online: 13 February 2018

(c) The Author(s) 2018. This article is an open access publication

\begin{abstract}
Background There is an unmet need to identify markers that predict the response to nivolumab in patients with non-smallcell lung cancer (NSCLC). The neutrophil-to-lymphocyte ratio (NLR) was recently recognized as an indicator of a poor prognosis in patients with various cancers. In the present study, we quantified the predictive impact of NLR in patients with NSCLC treated with nivolumab.

Methods We retrospectively analyzed 101 patients with advanced NSCLC treated with nivolumab at Kansai Medical University Hospital from December 2015 to December 2016. Patients were administered nivolumab at a dose of $3 \mathrm{mg} / \mathrm{kg}$ every 2 weeks. The predictive value of NLR for disease progression before treatment and 2 and 4 weeks after nivolumab treatment was assessed.

Results The median progression-free survival (PFS) of patients with an NLR of $<3$ before treatment was 3.4 months, whereas that of patients with an NLR of $\geq 3$ was 2.9 months $(p=0.484)$. The median PFS of patients with an NLR of $<3$ at 2 weeks after treatment was 5.3 months, whereas that of patients with an NLR of $\geq 3$ was 2.1 months $(p=0.00528)$. The median PFS of patients with an NLR of $<3$ at 4 weeks after treatment was 5.3 months, whereas that of patients with an NLR of $\geq 3$ was 2.0 months $(p=0.00515)$.

Conclusion The NLR at 2 and 4 weeks after treatment might be a useful marker for the prediction of the treatment response or disease progression in patients with advanced NSCLC receiving nivolumab.
\end{abstract}

Keywords Neutrophil-to-lymphocyte ratio (NLR) $\cdot$ Nivolumab $\cdot$ Non-small-cell lung cancer (NSCLC) $\cdot$ Progression-free survival (PFS) · Immune-related adverse event (irAE)

\section{Introduction}

Nivolumab is the first immune checkpoint inhibitor to be approved for relapse and refractory non-small-cell lung cancer (NSCLC) and has been the mainstay of treatment for NSCLC since 2015. Pembrolizumab was recently approved for first-line treatment of NSCLC. Thus, the advent of immune checkpoint inhibitors has significantly changed the treatment of NSCLC. When using pembrolizumab, a

Aya Nakaya

nakaya1016@yahoo.co.jp

1 First Department of Internal Medicine, Kansai Medical University, 2-5-1, Shin-machi, Hirakata, Osaka 573-1010, Japan programmed death ligand 1 (PD-L1) expression level of $>50 \%$ is essential [1]. However, it has been reported that whether the PD-L1 expression level over 1, 5, and $10 \%$ or not is related with the efficacy of nivolumab in patients with NSCLC [2, 3]. No reliable biomarker for prediction of the clinical outcome after nivolumab treatment has yet been established in daily clinical practice. Nivolumab is only effective in a portion of patients with NSCLC, and it is very expensive. Identifying biomarkers that predict treatment efficacy and thereby allow appropriate patient selection for these treatments is a crucial topic of ongoing research.

Inflammation is widely recognized to play an integral role in both the development and propagation of various cancers. Increased systemic inflammation is associated with a poorer prognosis in patients with cancer. The neutrophil-to-lymphocyte ratio (NLR), calculated as the absolute neutrophil count 
divided by the absolute lymphocyte count within the peripheral blood, has been shown to correlate with the prognosis of various malignancies [4-7]. The NLR has been evaluated in patients with both localized and advanced NSCLC and appears to be prognostic in these patient populations [8-10]. Furthermore, several reports have indicated that the NLR might predict the effects of cancer immune checkpoint inhibitors in patients with melanoma $[11,12]$.

The purpose of the present study was to determine whether the NLR is associated with the response to nivolumab therapy in patients previously treated for advanced NSCLC.

\section{Patients and methods}

We conducted a retrospective study of patients with NSCLC who were treated with single-agent nivolumab after platinum failure from January 2015 to December 2016 at Kansai Medical University Hospital, Osaka, Japan. Eligible patients had NSCLC with a history of platinum failure. Previously untreated patients were excluded from the study. Nivolumab $\left(3 \mathrm{mg} / \mathrm{m}^{2}\right)$ was administered every 2 weeks until the occurrence of disease progression, unacceptable toxicity, withdrawal, or death. NLR was evaluated before treatment and 2 and 4 weeks after the first treatment. We categorized the patients into two groups according to an NLR cut-off value of 3 . This cutoff was previously validated as being associated with inferior overall survival (OS) in patients with lung cancer in the largest such study to date [13]. This study was conducted in accordance with the Declaration of Helsinki and the requirements of the institution's review board.

\section{Study assessments}

Patient treatment responses were evaluated according to the Response Evaluation Criteria in Solid Tumors using wholebody computed tomography performed every $8-12$ weeks. However, because of the possibility of pseudo-progression in patients considered to have progressive disease, tumor size was carefully evaluated with reference to the guidelines for the Evaluation of Immune Therapy Activity in Solid Tumors [14]. The evaluation of toxicity was based on the Common Toxicity Criteria for Adverse Events, version 4.0 [15]. Adverse events (AEs) were evaluated for 12 weeks. Immune-related AEs (irAEs) were defined as rash, diarrhea, colitis, thyroid disorder, hepatitis, arthritis, and other conditions [16].

\section{Statistical analysis}

Progression-free survival (PFS) was defined as the time from the start of single-agent nivolumab treatment to objective disease progression. OS was calculated from the start of single-agent nivolumab treatment until the time of death or the last clinical follow-up. Survival curves were generated using the Kaplan-Meier method, and differences were evaluated using the log-rank test. Multivariate Cox-proportional hazards models were used to determine whether the patient baseline characteristics and/or NLR were associated with PFS. All statistical tests were two-sided. Statistical significance was defined as $p<0.05$, and $95 \%$ confidence intervals were calculated. All statistical analyses were performed using EZR (Saitama Medical Center, Jichi Medical University, Saitama, Japan), which is a graphical user interface for R version 2.13.0 (The R Foundation) [17]. Specifically, EZR is a modified version of R Commander (version 1.6-3), which adds statistical functions frequently used in biostatistics.

\section{Results}

\section{Patients}

The clinical characteristics of the patients $(n=101$; median age, 69 years, range $45-84$ years; $77 \%$ male) included in this study are shown in Table 1. Patients were diagnosed with either squamous cell carcinoma (37\%) or non-squamous cell carcinoma (63\%). Among all patients with adenocarcinoma, $10 \%$ had an epidermal growth factor receptor mutation and

Table 1 Patients' characteristics

\begin{tabular}{ll}
\hline No. of patients & 101 \\
\hline Median age (y/o), range & $69(45-84)$ \\
Male sex (\%) & 77 \\
ECOG PS(\%) & 84 \\
$0-1$ & 16 \\
$2 \leq$ & \\
Smoking history (\%) & 84 \\
Yes & 16 \\
No & \\
Histology (\%) & 37 \\
Squamous & 63 \\
Non-squamous & \\
Targetable driver mutation (\%) & 10 \\
EGFR & 3 \\
ALK & \\
No. of prior systemic Tx (\%) & 18 \\
1 & 28 \\
2 & 55 \\
$3 \leq$ &
\end{tabular}

EGOG Eastern Cooperative Oncology Group, $P S$ performance status, $E G F R$ epidermal growth factor receptor, $A L K$ anaplastic lymphoma kinase, $T x$ treatment 
$3 \%$ had the echinoderm microtubule-associated protein-like 4-anaplastic lymphoma kinase (EML4-ALK) fusion gene. One, two, and more than three previous regimens had been administered to 18,28 , and $55 \%$ of patients, respectively. The median follow-up period was 8.9 months.

\section{Treatment response and survival}

One percent of patients achieved a complete response, 25\% achieved a partial response, 35\% had stable disease, and 39\% had progressive disease (Fig. 1). Median PFS and OS were 3.2 (Fig. 2a) and 17.0 months (Fig. 2b), respectively.

\section{Relationship between NLR and PFS}

We evaluated NLR before treatment and 2 and 4 weeks after treatment to determine its association with PFS. The median PFS of patients with an NLR of $<3$ before treatment was 3.4 months, whereas that of patients with an NLR of $\geq 3$ was 2.9 months $(p=0.484)$ (Fig. 3a). The median PFS of patients with an NLR of $<3$ at 2 weeks after initial treatment was 5.3 months, whereas that of patients with an NLR of $\geq 3$ was 2.1 months ( $p=0.00528$ ) (Fig. 3b). The median PFS of patients with an NLR of $<3$ at 4 weeks after initial treatment was 5.3 months, whereas that of patients with an NLR of $\geq 3$ was 2.0 months ( $p=0.00515$ ) (Fig. 3c). We tried to clarify the relationship between the number of prior chemotherapy regimens and NLR. Although with patients with 1-2 prior lines, NLR did not reveal a strong predictive power for PFS (before treatment/2 weeks after initial treatment/4 weeks after initial treatment: $p=0.49 / 0.291 / 0.0875$ ). For patients with $\geq 3$ prior lines, NLR was statistically

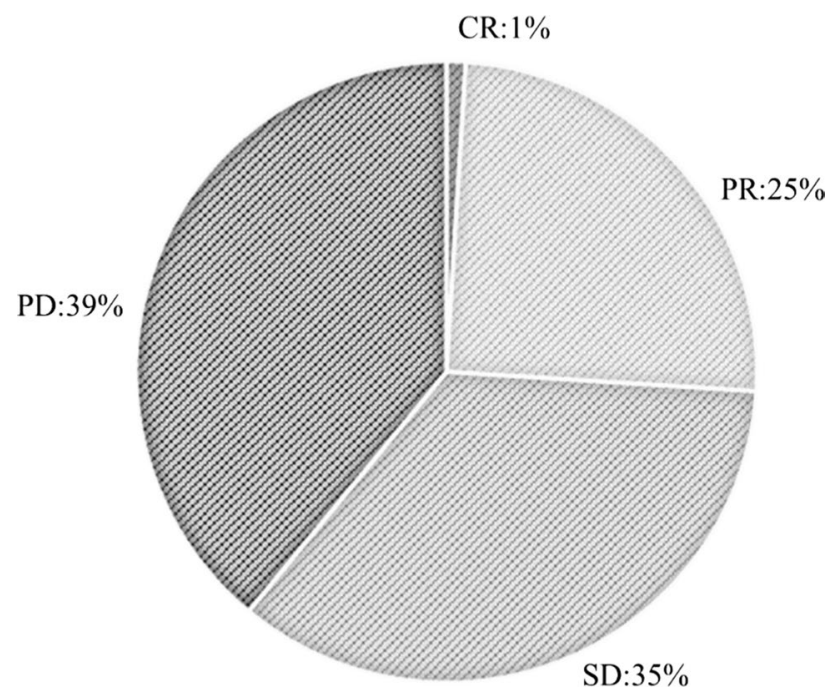

Fig. 1 Patients' treatment response rates. The patients' treatment responses were as follows: $1 \%$, complete response; $25 \%$, partial response; $35 \%$, stable disease; and 39\%, progressive disease
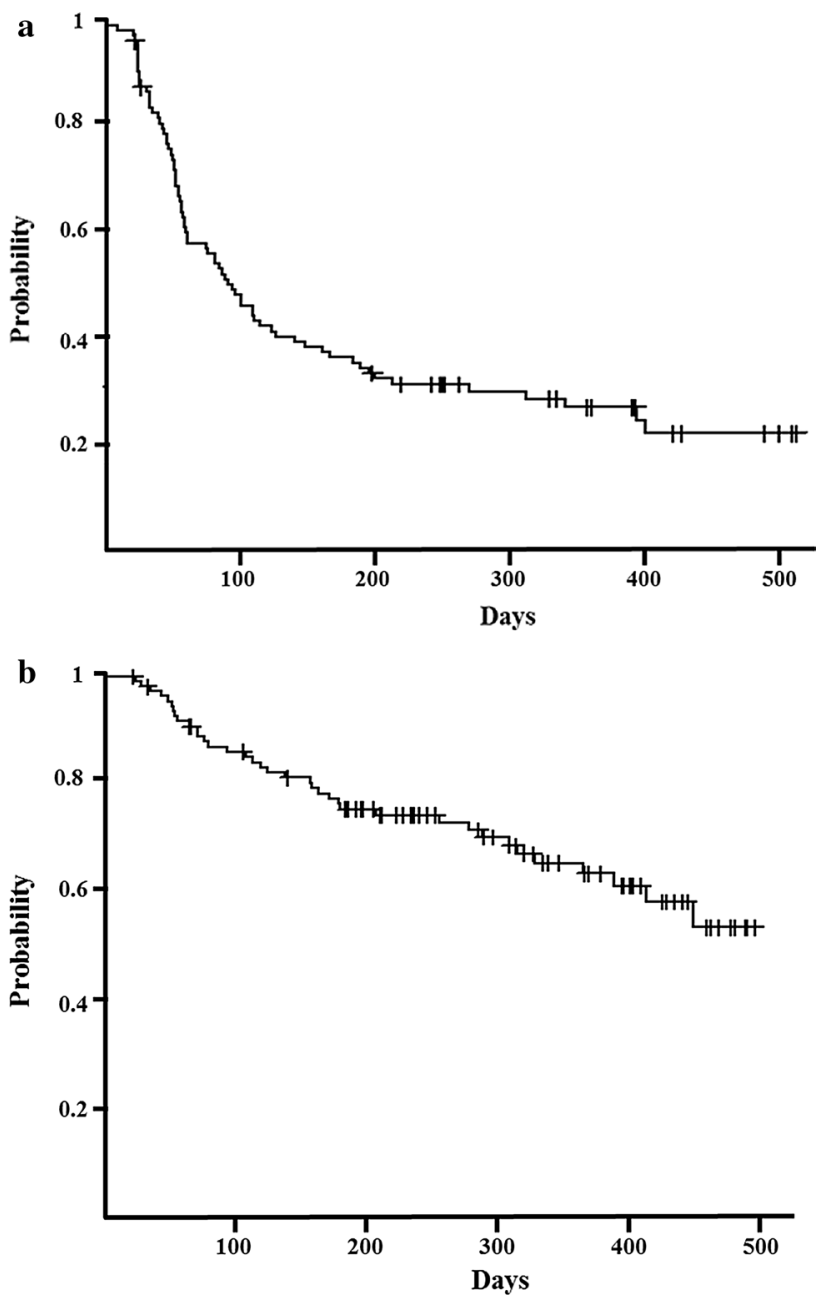

Fig. 2 Kaplan-Meier survival analysis. a Median progression-free survival, 3.2 months (range 2.1-4.1 months). b Median overall survival, 17.0 months (range 12.4 months-not available)

a useful tool to predict PFS (before/2 weeks/4 weeks: $p=0.478 / 0.00225 / 0.0104)$ (data not shown).

\section{Frequency of AEs}

The frequencies of AEs and irAEs are shown in Tables 2 and 3. The most common AEs (any grade) were fatigue $(n=26)$, appetite loss/nausea $(n=17)$, and rash $(n=17)$. Severe AEs (grade $>3)$ were anemia $(n=4)$, liver dysfunction $(n=2)$, and thyroid disorder $(n=2)$. The most common irAEs were rash $(n=15)$, liver dysfunction $(n=2)$, and thyroid dysfunction $(n=2)$.

Patients with irAEs $(n=40)$ had better PFS than patients without irAEs $(n=61)$, although the difference was not statistically significant (median, 4.4 vs. 2.1 months, respectively; $p=0.0516$ ) (Fig. 4a). Likewise, PFS between patients with AEs $(n=61)$ and without AEs $(n=40)$ was not 

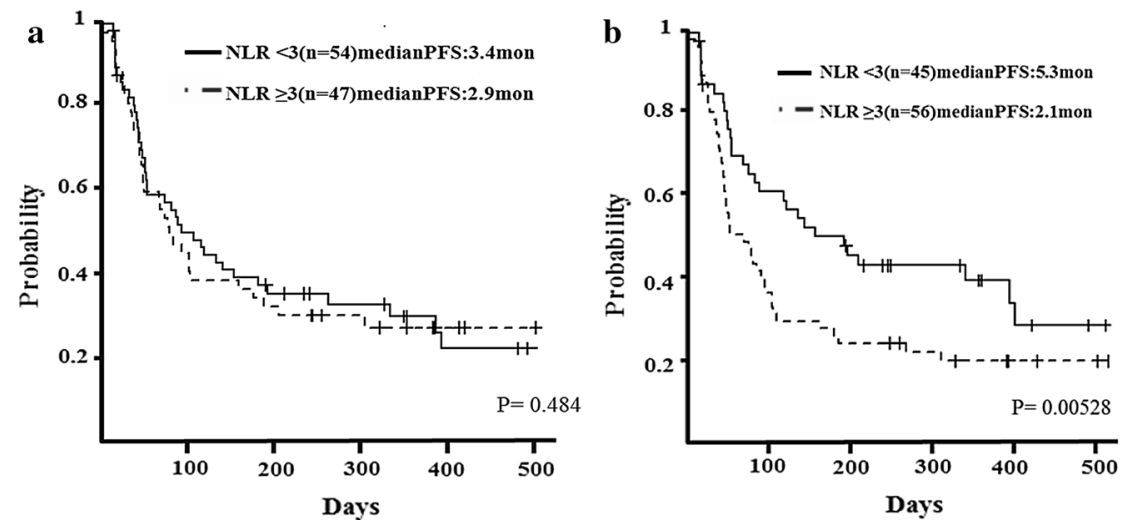

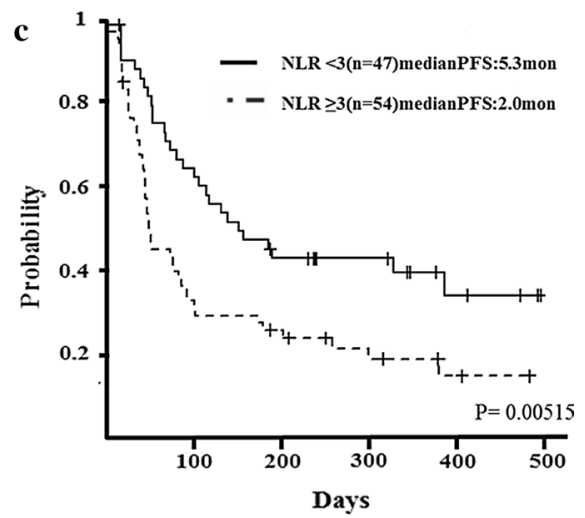

Fig. 3 Relationship between neutrophil-to-lymphocyte ratio (NLR) and progression-free survival (PFS). a Median PFS of patients with an NLR of $<3$ before treatment was 3.4 months, whereas that of patients with an NLR of $\geq 3$ was 2.9 months $(p=0.484)$. b Median PFS of patients with an NLR of $<3$ at 2 weeks after the initial treat-

Table 2 Adverse events

\begin{tabular}{lcl}
\hline Adverse events & Any grade $(n)$ & Grade \\
& & $3-4(n)$ \\
\hline Fatigue & 26 & 1 \\
Appetite loss/nausea & 17 & 0 \\
Rash & 15 & 1 \\
Anemia & 9 & 4 \\
Liver dysfunction & 8 & 2 \\
Thyroid disorder & 8 & 2 \\
Interstitial lung disease & 6 & 1 \\
Edema & 5 & 0 \\
Diarrhea & 4 & 0 \\
Mucositis & 4 & 1 \\
Renal dysfunction & 3 & 0 \\
Fever & 3 & 0 \\
Others & 17 & 8 \\
\hline
\end{tabular}

significantly different (median, 2.1 vs. 3.4 months, respectively; $p=0.665$ ) (Fig. 4b).

\section{Multivariate analysis}

We also investigated other factors that may be associated with the outcome of nivolumab treatment, such as age of $>75$ years, sex, number of prior chemotherapy treatments, histology, elevation of C-reactive protein, irAEs, and NLR at 2 and 4 weeks after treatment. Multivariate analysis showed that more than three lines of chemotherapy were associated with significantly worse PFS. In contrast, patients with irAEs had significantly better PFS. An NLR of $<3$ at neither 2 nor 4 weeks after treatment was associated with significantly better PFS in the multivariate analysis (Table 4). ment was 5.3 months, whereas that of patients with an NLR of $\geq 3$ was 2.1 months $(p=0.00528)$. c Median PFS of patients with an NLR of $<3$ at 4 weeks after the initial treatment was 5.3 months, whereas that of patients with an NLR of $\geq 3$ was 2.0 months $(p=0.00515)$

Table 3 Immune-related adverse events

\begin{tabular}{ll}
\hline Immune-related adverse events & Any grade $(n)$ \\
\hline Skin & \\
Rash & 15 \\
Hepatic & 8 \\
$\quad$ Liver dysfunction & \\
Endocrine & 8 \\
Thyroid disorder & 2 \\
Adrenal disorder & \\
Gastro-intestinal & 4 \\
Diarrhea & 4 \\
Mucositis & \\
Pulmonary & 6 \\
Interstitial lung disease & \\
Renal & 3 \\
Renal dysfunction & \\
Others & 2 \\
Hypoalbuminemia & 5 \\
Edema & 1 \\
Thrombocytopenia & 1 \\
Pleural effusion & 1 \\
Parotitis & \\
\hline
\end{tabular}

\section{Discussion}

Our study revealed that an NLR of $<3$ at 2 and 4 weeks after nivolumab treatment might be an independent prognostic biomarker in patients in patients with advanced NSCLC. This is the first study to evaluate the power of NLR to predict the outcome of nivolumab treatment in an Asian cohort. Bagley et al. recently found that 

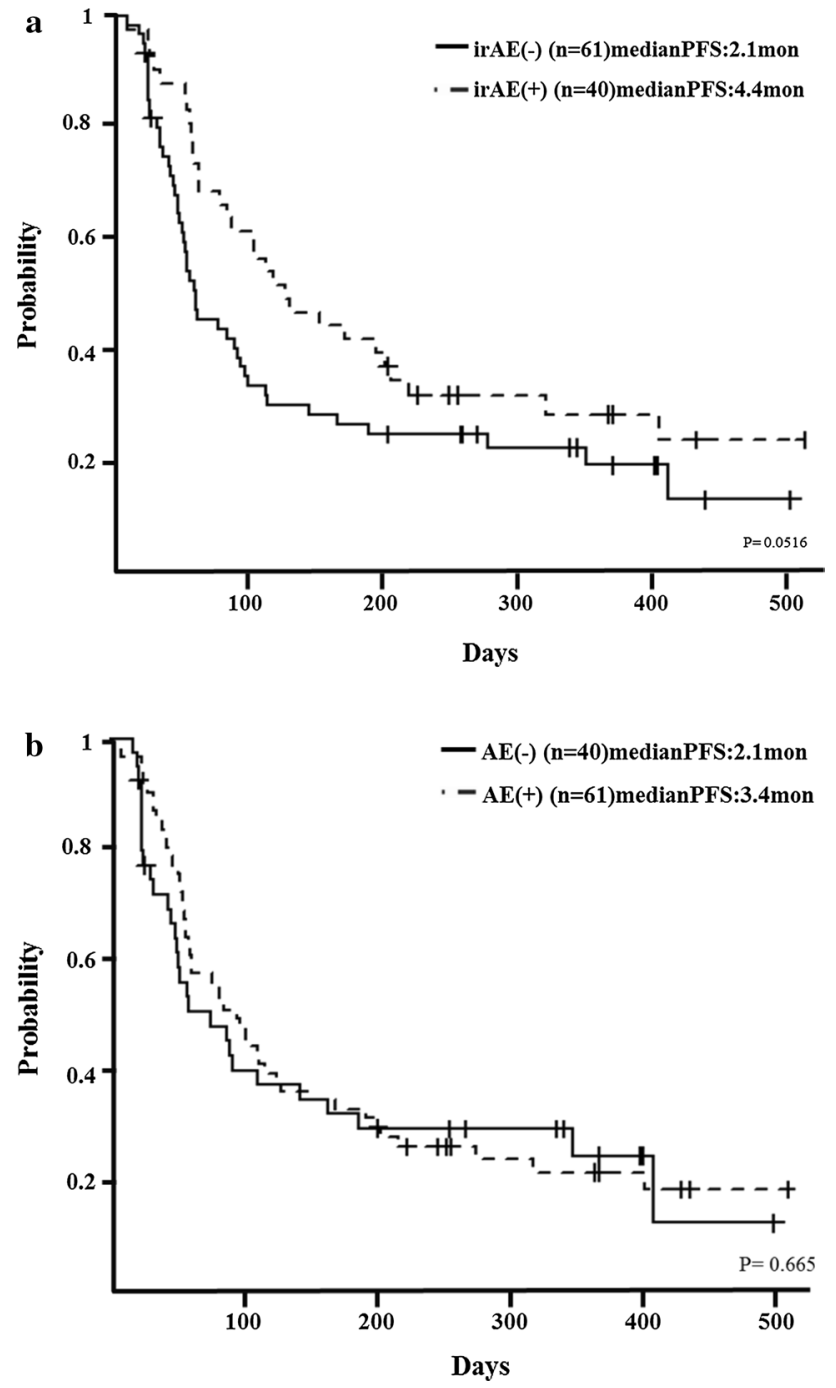

Fig. 4 Relationship between adverse events (AEs) and progressionfree survival (PFS). a Median PFS of patients with immune-related AEs was 4.4 months, whereas that of patients without immunerelated AEs was 2.1 months $(p=0.0516)$. b Median PFS of patients with AEs was 2.1 months, whereas that of patients without AEs was 3.4 months $(p=0.665)$

pretreatment NLR was associated with inferior OS and PFS in patients with lung cancer treated with nivolumab [18]. However, these patients had been treated with several regimens; thus, pretreatment NLR is presumed to have been affected by the previous treatment. In the present study, pretreatment NLR was not associated with PFS.

In recent years, it has become increasingly apparent that cancer-associated inflammation is a key determinant of disease progression and survival in patients with many types of solid tumors [19]. Because immune checkpoint inhibitors enhance antitumor immunity by blocking negative regulators of T-cell function [20], it is plausible that alterations in the relative proportions of circulating lymphocytes could
Table 4 Multivariate analysis

\begin{tabular}{|c|c|c|c|}
\hline & Odds ratio & $95 \% \mathrm{CI}$ & $p$ \\
\hline \multicolumn{4}{|l|}{ Age } \\
\hline$\geq 75$ years & 0.691 & $0.3876-1.2320$ & 0.2104 \\
\hline$<75$ years & 1 & & \\
\hline \multicolumn{4}{|l|}{ Sex } \\
\hline Male & 0.6541 & $0.3820-1.1200$ & 0.1218 \\
\hline Female & 1 & & \\
\hline \multicolumn{4}{|l|}{ Prior line } \\
\hline $3 \leq$ & 2.012 & $1.2220-3.3130$ & 0.006019 \\
\hline $1-2$ & 1 & & \\
\hline \multicolumn{4}{|l|}{ Histology } \\
\hline Squamous & 1.36 & $0.8401-2.2000$ & 0.2111 \\
\hline Non-squamous & 1 & & \\
\hline \multicolumn{4}{|l|}{$\mathrm{C}$-reactive protein } \\
\hline$\geq 1$ & 0.973 & $0.5521-1.7150$ & 0.9246 \\
\hline$<1$ & 1 & & \\
\hline \multicolumn{4}{|l|}{ irAEs } \\
\hline+ & 0.5785 & $0.3404-0.9831$ & 0.04309 \\
\hline- & 1 & & \\
\hline \multicolumn{4}{|l|}{ NLR 2 weeks } \\
\hline$\geq 3$ & 1.368 & $0.7600-2.4630$ & 0.2959 \\
\hline$<3$ & 1 & & \\
\hline \multicolumn{4}{|l|}{ NLR 4 weeks } \\
\hline$\geq 3$ & 1.595 & $0.9278-2.7430$ & 0.0912 \\
\hline$<3$ & 1 & & \\
\hline
\end{tabular}

irAE immune-related adverse event, $N L R$ neutrophil-to-lymphocyte ratio

influence the efficacy of immune checkpoint inhibitors. Inflammatory cytokines and chemokines can be produced by both the tumor and associated host cells such as leukocytes, contributing to malignant progression [21]. As an indicator of inflammation and tumor immune response, NLR plays a pivotal role in various cancers. However, the exact mechanism that explains the poor survival outcomes of patients with cancer who have a high NLR remains unclear.

Neutrophils can produce various chemokines and cytokines and suppress the immune activity of lymphocytes and natural killer cells. Tumor-associated neutrophils are derived from peripheral neutrophils and have been proposed as key mediators in tumor progression specifically because they induce genetic instability, promote tumor growth, stimulate angiogenesis, and favor the invasive behavior of cancer cells [22].

The importance of lymphocytes has been highlighted in several studies in which increased infiltration of lymphocytes into tumors was associated with a better response to cytotoxic treatment and progression in patients with cancer [23-25]. Lymphocytes play a crucial role in host cell-mediated immune regulation, which is important for destruction 
of residual malignant cells [26]. It is widely believed that tumor-infiltrating lymphocytes (TILs) are associated with better clinical outcomes in patients with cancer [27].

The expression of PD-L1 and the presence of TILs in melanoma influence the therapeutic effect of immune checkpoint inhibitors [28]. Positivity of both PD-L1 and TILs is the most effective status for efficacy of immune checkpoint inhibitors. We therefore assumed that NLR might reflect the presence of TILs and predict the therapeutic effect.

We clarified the relationship between the number of prior chemotherapy regimens and NLR. Our result revealed that NLR at 2 and 4 weeks after the initial treatment was a useful predictive factor for PFS using nivolumab in patients with $\geq 3$ prior lines, which are known to be related to poor outcome.

Although the mechanism is unknown, NLR reflects the balance between inflammation and immunoreaction and has long been used as an effective prognostic biomarker in patients with various cancers, including lung cancer [18, 29-31].

Interestingly, irAE-positive patients had better PFS in the present study. In a previous study, irAE-positive patients with melanoma treated with nivolumab had better OS [27]. This is the first study to evaluate the relationship between irAEs and PFS in patients with NSCLC treated with nivolumab. IrAEs might be a prospective factor of nivolumab efficacy in patients with NSCLC.

This study has a few limitations. First, the most effective cut-off value of the NLR is unknown. In our cohort, the median NLR was 2.6. We considered whether to set a cutoff of 2 or 3 . With a cutoff of 2 or more, the number of patients over the cutoff of 2 is about $70 \%$ and there is a bias. On the other hand, with a cutoff of 3 and above, balance of the number of patients below and above the cutoff is about fifty-fifty. The median PFS of patients with an NLR of $<2$ before treatment $(n=27)$ was 5.0 months, whereas that of patients with an NLR of $\geq 2$ was 2.9 months $(n=71)$ $(p=0.258)$. The median PFS of patients with an NLR of $<2$ at 2 weeks after the initial treatment $(\mathrm{n}=16)$ was 7.0 months, whereas that of patients with an NLR of $\geq 2(n=82)$ was 2.9 months $(p=0.347)$. The median PFS of patients with an NLR of $<2$ at 4 weeks after the initial treatment $(n=19)$ was 7.1 months, whereas that of patients with an NLR of $\geq 2$ $(n=19)$ was 2.7 months $(p=0.024)$. There was no statistically significant difference with a cutoff of 2 . Therefore, we adopted a cutoff of 3 in the current study. Furthermore, we used a cutoff of 3 according to the largest cohort study performed to date [13]. However, various studies of the usefulness of NLR have used different cut-off values, and the methods of selecting these NLR cutoffs were unclear in many studies. Prospective studies are needed to verify the adaptive cut-off value. Second, NLR at 2 and 4 weeks after the initial treatment was significantly associated with PFS; however, we failed to identify a pretreatment biomarker. Because nivolumab is an expensive agent, an effective biomarker must be identified before we decide to use this agent. Although irAEs are an efficient biomarker, they cannot serve as a pretreatment biomarker. Thus, another biomarker with which to predict the efficacy of nivolumab before treatment is needed. Furthermore, in the multivariate analysis, NLR was not a prognostic factor for PFS. This might have been due to the number of patients in this study, which may have been too small to reveal a significant difference in NLR in the multivariate analysis. Finally, we did not use immunerelated response criteria, because this was not a prospective trial and most physicians are not yet familiar with such criteria. We were also unable to examine the expression of PD-L1 before treatment because of the limitation associated with patient insurance policies.

In conclusion, measurement of NLR during nivolumab treatment might be a simple and useful early biomarker in patients with advanced NSCLC. However, its use needs to be evaluated in a larger prospective cohort study.

\section{Compliance with ethical standards}

Conflict of interest T. Kurata received honoraria from Ono Pharmaceutical and Bristol-Myers Squibb. The other authors disclosed no potential conflicts of interest.

Open Access This article is distributed under the terms of the Creative Commons Attribution 4.0 International License (http://creativecommons.org/licenses/by/4.0/), which permits unrestricted use, distribution, and reproduction in any medium, provided you give appropriate credit to the original author(s) and the source, provide a link to the Creative Commons license, and indicate if changes were made.

\section{References}

1. Herbst RS, Baas P, Kim DW et al (2016) Pembrolizumab versus docetaxel for previously treated, PD-L1-positive, advanced nonsmall-cell lung cancer (KEYNOTE-010): a randomised controlled trial. Lancet 387:1540-1550

2. Brahmer J, Reckamp KL, Baas P et al (2015) Nivolumab versus docetaxel in advanced squamous-cell non-small-cell lung cancer. N Engl J Med 373:123-135

3. Borghaei H, Paz-Ares L, Horn L et al (2015) Nivolumab versus docetaxel in advanced nonsquamous non-small-cell lung cancer. N Engl J Med 373:1627-1639

4. Templeton AJ, McNamara MG, Šeruga B et al (2014) Prognostic role of neutrophil-to-lymphocyte ratio in solid tumors: a systematic review and meta-analysis. J Natl Cancer Inst 106:124. https ://doi.org/10.1093/jnci/dju124

5. Luo G, Guo M, Liu Z et al (2015) Blood neutrophil-lymphocyte ratio predicts survival in patients with advanced pancreatic cancer treated with chemotherapy. Ann Surg Oncol 22:670-676

6. Noh H, Eomm M, Han A (2013) Usefulness of pretreatment neutrophil to lymphocyte ratio in predicting disease-specific survival in breast cancer patients. J Breast Cancer. 16:55-59 
7. Dalpiaz O, Ehrlich GC, Mannweiler S et al (2014) Validation of pretreatment neutrophil-lymphocyte ratio as a prognostic factor in a European cohort of patients with upper tract urothelial carcinoma. BJU Int 114:334-339

8. Cedrés S, Torrejon D, Martínez A et al (2012) Neutrophil to lymphocyte ratio (NLR) as an indicator of poor prognosis in stage IV non-small cell lung cancer. Clin Transl Oncol 14:864-869

9. Forget P, Machiels JP, Coulie PG et al (2013) Neutrophil:lymphocyte ratio and intraoperative use of ketorolac or diclofenac are prognostic factors in different cohorts of patients undergoing breast, lung, and kidney cancer surgery. Ann Surg Oncol 20(Suppl 3):S650-S660

10. Jafri SH, Shi R, Mills G (2013) Advance lung cancer inflammation index (ALI) at diagnosis is a prognostic marker in patients with metastatic non-small cell lung cancer (NSCLC): a retrospective review. BMC Cancer 13:158. https://doi. org/10.1186/1471-2407-13-158

11. Ferrucci PF, Gandini S, Battaglia A et al (2015) Baseline neutrophil-to-lymphocyte ratio is associated with outcome of ipilimumab-treated metastatic melanoma patients. Br J Cancer 112:1904-1910

12. Nakamura Y, Kitano S, Takahashi A et al (2016) Nivolumab for advanced melanoma: pretreatment prognostic factors and early outcome markers during therapy. Oncotarget 7:77404-77415

13. Akinci Ozyurek B, Sahin Ozdemirel T, Buyukyaylaci Ozden S et al (2017) Prognostic value of the neutrophil to lymphocyte ratio (NLR) in lung cancer cases. Asian Pac J Cancer Prev 18:1417-1421

14. Wolchok JD, Hoos A, O'Day S et al (2009) Guidelines for the evaluation of immune therapy activity in solid tumors: immunerelated response criteria. Clin Cancer Res 15:7412-7420

15. http://ctep.cancer.gov/protocolDevelopment/electronic_applicatio ns/ctc.htm\#ctc_40. Accessed on Aug 162017

16. Weber JS, Kähler KC, Hauschild A (2012) Management of immune-related adverse events and kinetics of response with ipilimumab. JCO 30:2691-2697

17. Kanda Y (2015) Statistical analysis using freely available "EZR (Easy R)" software. Rinsho Ketsueki 56:2258-2266

18. Bagley SJ, Kothari S, Aggarwal C et al (2017) Pretreatment neutrophil-to-lymphocyte ratio as a marker of outcomes in nivolumab-treated patients with advanced non-small-cell lung cancer. Lung Cancer 106:1-7
19. Hanahan D, Weinberg RA (2011) Hallmarks of cancer: the next generation. Cell 144:646-674

20. Pardoll DM (2012) The blockade of immune checkpoints in cancer immunotherapy. Nat Rev Cancer 12:252-264

21. Balkwill F, Mantovani A (2001) Inflammation and cancer: back to Virchow? Lancet 357:539-545

22. Galdiero MR, Garlanda C, Jaillon S et al (2013) Tumor associated macrophages and neutrophils in tumor progression. J Cell Physiol 228:1404-1412

23. Loi S, Sirtaine N, Piette F et al (2013) Prognostic and predictive value of tumor-infiltrating lymphocytes in a phase III randomized adjuvant breast cancer trial in node-positive breast cancer comparing the addition of docetaxel to doxorubicin with doxorubicinbased chemotherapy: BIG 02-98. J Clin Oncol 31:860-867

24. Gooden MJ, de Bock GH, Leffers N et al (2011) The prognostic influence of tumour-infiltrating lymphocytes in cancer: a systematic review with meta-analysis. Br J Cancer 105:93-103

25. Denkert C, Loibl S, Noske A, Roller M et al (2010) Tumor-associated lymphocytes as an independent predictor of response to neoadjuvant chemotherapy in breast cancer. J Clin Oncol 28:105-113

26. Sarraf KM, Belcher E, Raevsky E et al (2009) Neutrophil/lymphocyte ratio and its association with survival after complete resection in non-small cell lung cancer. J Thorac Cardiovasc Surg 137:425-428

27. Zhang L, Conejo-Garcia JR, Katsaros D et al (2003) Intratumoral $\mathrm{T}$ cells, recurrence, and survival in epithelial ovarian cancer. $\mathrm{N}$ Engl J Med 348:203-213

28. Teng MW, Ngiow SF, Ribas A et al (2015) Classifying cancers based on T-cell infiltration and PD-L1. Cancer Res 75:2139-2145

29. Byun SS, Hwang EC, Kang SH et al (2016) Prognostic significance of preoperative neutrophil-to-lymphocyte ratio in nonmetastatic renal cell carcinoma: a large, multicenter cohort analysis. Biomed Res Int 2016:5634148

30. Peng B, Wang YH, Liu YM et al (2015) Prognostic significance of the neutrophil to lymphocyte ratio in patients with non-small cell lung cancer: a systemic review and meta-analysis. Int J Clin Exp Med 8:3098-3106

31. Freeman-Keller M, Kim Y, Cronin H, Richards A et al (2016) Nivolumab in resected and unresectable metastatic melanoma: characteristics of immune-related adverse events and association with outcomes. Clin Cancer Res 22:886-894 